\title{
Fabrication of Polymer Microfiber through Direct Drawing and Splicing of Silica Microfiber via Vapor Spray and Flame Treatment
}

\author{
Y. S. Ong ${ }^{1,2}$, W. Kam $^{1,2}$, S.W. Harun ${ }^{2}$, and *R.Zakaria ${ }^{1}$
}

\begin{abstract}
We report the simple fabrication technique of polymer microfiber with high surface smoothness and length uniformity using a molten polymethyl methacrylate (PMMA) in conjunction with direct drawing technique. With the aid of fabricated polymer microfiber, we successfully demonstrate a new splicing technique via two steps processes. The fabricated microfiber with diameter of $1.5 \mu \mathrm{m}$ and length of $3 \mathrm{~mm}$ has been used in splicing the silica microfibers based on solvent vapor spray and flame treatment process. The permanent splicing has been achieved after silica fiber fused with flame and polymer fiber is removed. We were able to obtain an average splicing loss of about $-0.69 \mathrm{~dB}$. Using the same technique, we also demonstrated the splicing for ring resonator. The splicing technique demonstrated here promises high potential for robust optical integration of microfibers for various functional circuits and devices.
\end{abstract}

Keywords—optical fiber, sensors

\section{Introduction}

Microfibers have gained a tremendous interest in recent years as promising components for sub wavelength wave guiding and nanophotonics devices. They have been drawn and demonstrated by flame-heated silica fiber [1] and bulk glasses [2] method. The method provides an easy and cheap photonic wires manufacturing technique, with constant temperature distribution is required in the drawing region. Later, flamebrushing technique was proposed to fabricate silica microfibers $[3,4]$, which can be used to assemble a variety of functional structures, such as resonators [5], interferometers [6, 7], filters [8], sensors [9, 10], and lasers [11]. To maintain the functional shape or geometry of the assembled structures, several approaches have been reported including van der Waals attraction [7, 12, 13], substrate-supported friction [14, 15], laserheated fusion splicing [16, 17]and splicing via polymer nanowires [18]. Although the van der Waals attraction and substrate-supported friction are easy to realize, they are fragile to environmental disturbance and impossible to sustain in liquid. Laser-heated fusion splicing offers much higher robustness; however it is limited to glass microfibers and requires a complicated experimental system. For splicing via polymer nanowires, amount of polymers are quite critical and have to control well in order to reduce the splicing loss and they are fragile to higher temperature and hard to sustain in solvent. Polymer optical microfibers may play a key role in several rapid developing areas for broadband communications and also for sensing purposes because of their chemical specificities, low cost, mechanical flexibility, tunable properties, ease process ability and integration [19].

${ }^{1}$ Department of Physics, University of Malaya, 50603 Kuala Lumpur, Malaysia

${ }^{2}$ Photonic Research Centre, Faculty of Science, University of Malaya, 50603 Kuala Lumpur, Malaysia
In this paper, we demonstrate an eco-friendly and simple drawing method of fabricating polymer microfibers. This drawing method somewhat similar with dissolved polymer drawing [23] but required no solvent and polymer state is manipulated through temperature control. Polymethyl-methacrylate (PMMA) polymer used in this work have many interesting properties such as lower lost at visible light and high refractive index, which provides a good optical confinement.

\section{Experimental Method}

Using direct drawing technique, polymer microfibers with diameter of $1.5 \mu \mathrm{m}$ and length $3 \mathrm{~mm}$ were fabricated by one-step process. Polymethyl methacrylate (PMMA) is selected as the polymer wave-guiding material in this experiment because of its high mechanical strength, good dimensional stability, good weather resistance and natural transparent above deep ultraviolet. A hot plate is used to melt PMMA and keep temperature at constant during the fiber drawing. For this polymer it is important to maintain desirable viscosity level, which means temperature of heating plate should be controlled within the temperature range between glass transition temperature and melting temperature of polymer. As the glass transition temperature ( $\mathrm{Tg}$ ) of PMMA ranges from 85 to $165^{\circ} \mathrm{C}$, heating plate temperature was kept around $115^{\circ} \mathrm{C}$ during the fiber drawing. Firstly, a silica fiber with diameter about $125 \mu \mathrm{m}$ is being resembled and its tip is immersed into the molten PMMA.

\section{References}

[1] 1. Tong, L., et al., Subwavelength-diameter silica wires for lowloss optical wave guiding. Nature, 2003. 426(6968): p. 816-819.

[2] 2. Tong, L., et al., Photonic nanowires directly drawn from bulk glasses. Optics Express, 2006. 14(1): p. 82-87.

[3] 3. Brambilla, G., F. Xu, and X. Feng, Fabrication of optical fibre nanowires and their optical and mechanical characterisation. Electronics Letters, 2006. 42(9): p. 517-519.

[4] 4. Harun, S., et al., Fabrication of tapered fiber based ring resonator. Laser physics, 2010. 20(7): p. 1629-1631.

[5] 5. Xu, F. and G. Brambilla, Manufacture of 3-D microfiber coil resonators. Photonics Technology Letters, IEEE, 2007. 19(19): p. 1481-1483.

[6] 6. Li, Y. and L. Tong, Mach-Zehnder interferometers assembled with optical microfibers or nanofibers. Optics letters, 2008. 33(4): p. 303-305.

[7] 7. Sulaiman, A., et al., Microfiber Mach-Zehnder interferometer embedded in low index polymer. Optics \& Laser Technology, 2012. 44(4): p. 1186-1189. 